\title{
A rare case of uterus didelphys presented as secondary abdominal pregnancy
}

\author{
Neena Gupta ${ }^{1}$, Sachidanand Gupta ${ }^{3}$, Neetu Singh ${ }^{1}$, Arun Kumar Arya ${ }^{2}$, \\ Pavika Lal ${ }^{1}$, Monica Chauhan ${ }^{1}$
}

\begin{abstract}
${ }^{1}$ Department of Obstetrics \& Gynaecology, ${ }^{2}$ Department of Paediatrics, GSVM Medical College, Kanpur, UP, India ${ }^{3}$ Department of Anaesthesia, Rama Medical College, Kanpur, UP, India
\end{abstract}

Received: 21 October 2013

Accepted: 27 October 2013

\section{*Correspondence:}

Dr. Neena Gupta,

E-mail: neena.gupta2211@gmail.com

(C) 2013 Gupta $\mathrm{N}$ et al. This is an open-access article distributed under the terms of the Creative Commons Attribution Non-Commercial License, which permits unrestricted non-commercial use, distribution, and reproduction in any medium, provided the original work is properly cited.

\section{ABSTRACT}

Uterus didelphys is a rare type of uterine malformation with poor obstetric outcome. We report a rare case of ruptured uterus didelphys presenting as secondary abdominal pregnancy which was managed by removal of ruptured uterus along with the omentum to which placenta was attached. There should be a high degree of suspicion for proper management of such cases as these patients carry great morbidity and mortality if left undiagnosed and untreated.

Keywords: Uterus didelphys, Secondary abdominal pregnancy, Maternal morbidity, Maternal mortality

\section{INTRODUCTION}

An abdominal pregnancy is the rarest and most serious type of extrauterine gestation associated with high morbidity and mortality with the rise for death 7-8 times greater than tubal ectopic pregnancy and 50 times greater than from intrauterine pregnancy. ${ }^{1}$ Incidence of abdominal pregnancy varies from 1 in 372 to 1 in 9,714 live births and account for $1.4 \%$ of ectopic pregnancies. ${ }^{2}$ Secondary abdominal pregnancy result from tubal abortion or tubal rupture or less often from subsequent implantation within the abdomen after uterine rupture.

Spontaneous uterine rupture in pregnancy is a rare and often catastrophic complication with a high incidence of foetal and maternal morbidity and mortality. We report a rare case of secondary abdominal pregnancy after spontaneous uterine rupture of uterus didelphys at 19 weeks +6 days period of gestation.

\section{CASE REPORT}

Mrs. X age 30 yrs female G2P1+0 (FTNVD) with one living issue 3 years back presented with chief complaints of acute pain in lower abdomen since one day following 5 months amenorrhea during emergency. On admission her general condition was average, pulse rate-110/min, B.P.$90 / 60 \mathrm{~mm}$ of $\mathrm{Hg}$, cardiovascular and respiratory systems were within normal limits, abdomen was distended along with rebound tenderness. On per speculum-blood mixed discharge was present and on per vaginal examinationuterus size could not be assessed. All her routine investigations were sent which were normal except her $\mathrm{Hb}-5.3 \mathrm{gm} \%$. A transabdominal ultrasound was done in emergency which revealed an empty uterus with a single dead fetus of average maturity 19wks 6 days in the abdominal cavity. Therefore diagnosis of rupture uterus was made and patient was taken for exploratory laparotomy under general anaesthesia. Preoperative findings revealed hemoperitoneum of about 3 liters, dead fetus of about 5 months gestation in the peritoneal cavity 
with placental attachment to the omentum and uterus didelphys was present with rupture of one uterus. The ruptured uterus was removed along with removal of part of omentum to which placenta was attached. She received 3 units of blood transfusion and her intraoperative and post-operative period was uneventful and was discharged in satisfactory condition.

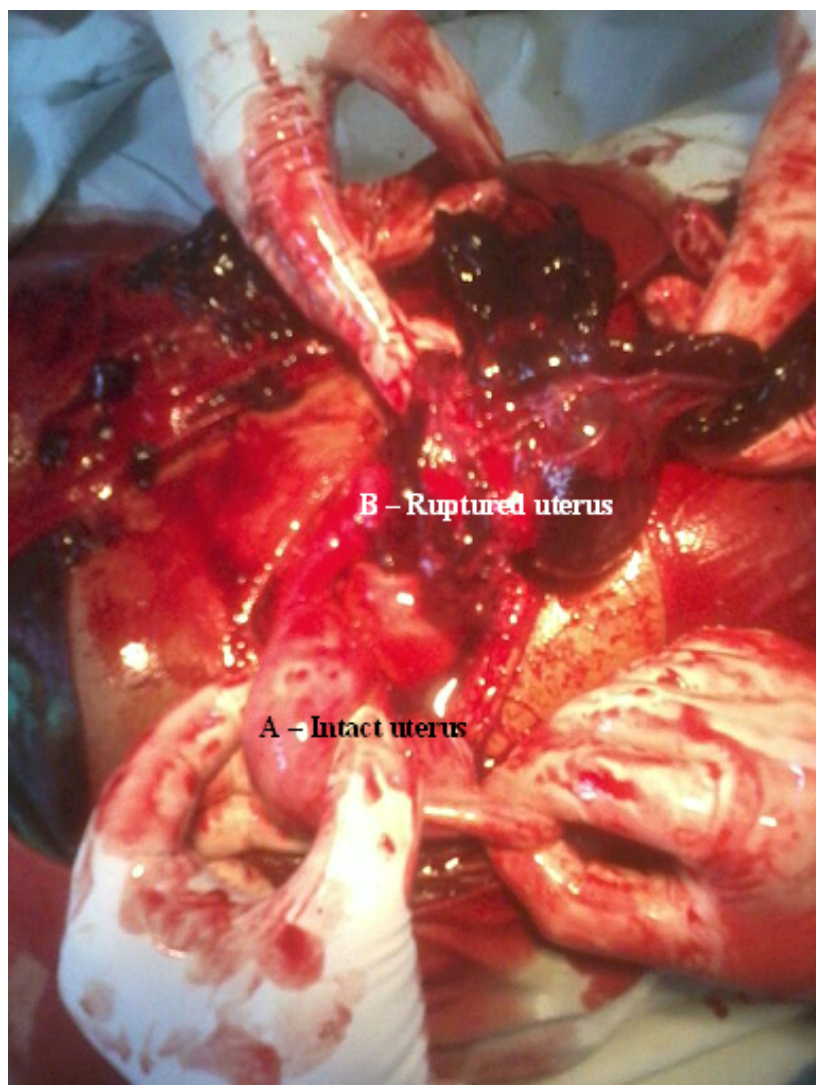

Figure 1: Uterus didelphys showing (A) Intact uterus and (B) Ruptured uterus.

\section{DISCUSSION}

The prevalence of uterine malformation is estimated to be $6.7 \%$ in the general population, slightly higher $(7.3 \%)$ in the infertility population, and significantly higher in a population of women with a history of recurrent miscarriages $(16 \%) .^{3}$ The uterus is formed during embryogenesis by the fusion of the two paramesonephric ducts (also called mullerian ducts). This process usually fuses the two mullerian ducts into a single uterine body but when both ducts fail to fuse, patient has a "double uterus" or uterus didelphys. This may be a condition with a double cervix and a vaginal partition, or the lower mullerian system fused into its unpaired condition. Double uterus has a poor obstetric outcome. It can be considered in patients of severe dysmenorrhoea with chronic pelvic pain, failure of intrauterine contraceptive devices, or of spontaneous abortion, still birth and in preterm birth. In such cases, the walls of the abnormal uteri tend to become abnormally thin as pregnancies advances, and the thickness can be inconsistent over different aspects of the myometrium. ${ }^{4}$

Recently 3-D ultrasonography has been advocated as an excellent non-invasive method to evaluate congenital uterine malformations. ${ }^{5}$ MRI has been used with success to diagnose abdominal pregnancy. ${ }^{6}$ The extent of placental invasion, its relationship to the pelvic organs and the vascular supply could be more closely visualised by magnetic resonance imaging. ${ }^{7}$

In very rare cases of uterine rupture the foetus may be extruded into the peritoneal cavity while the placenta remains functional within the uterus and the gestation continues as a uteroabdominal pregnancy. ${ }^{8}$ Abdominal pregnancies should be interrupted at diagnosis, as the potential for delivering of a healthy infant is poor and the risk of maternal complications is high. In our case secondary abdominal pregnancy was seen in association with uterus didelphys which makes it unique. A case of advanced secondary abdominal pregnancy through a small rent in rudimentary horn was reported by Desai et al. ${ }^{9}$ High index of suspicion is required in such cases and it usually involves the removal of the ruptured uterus as it was done in this case.

\section{CONCLUSION}

Uterine abnormalities, although rare can be encountered in pregnancy. Attempts should be made for early diagnosis to avoid maternal morbidity and mortality. There is a need to build capacity for making an antenatal diagnosis of such malformations in order to ensure appropriate management. ${ }^{10}$ However it still remains a diagnostic and therapeutic challenge when such patients come in acute emergency but with timely and proper intervention, life of the patient can be saved.

\section{REFERENCES}

1. Bouyer J, Fernandez H, et al. Sites of ectopic pregnancy: a 100 year population -based study of 1800 cases. Hum Reprod 2002; 17:3224-3230.

2. Atrash HK, Friede A, Hogue CJR. Abdominal pregnancy in the United States: frequency and maternal mortality. Obstet Gynecol 1987;69:333337.

3. Sotirios H. Saravelos, Karen A. Cocksedge and TinChiu Li. "Prevalence and diagnosis of congenital uterine anomalies in women with reproductive failure: a critical appraisal." Human Reproduction Update 2008 ; 14 (5): 415-29.

4. Nahum GG. Uterine anomalies, induction of labor, $\&$ uterine rupture. Obstet Gynaecol Nov 2005:106(5):1150-52.

5. Woelfer B., Salim R., Banerjee S., Elson J., Regan L., Jutkovic D. Reproductive outcomes in women with congenital uterine anomalies detected by threedimensional ultrasound screening. Obstet Gynecol, 2001; 98(6): 1099-103. 
6. Dahiya K, Sharma D. Advanced abdominal pregnancy; A diagnostic and management dilemma. J Gynecol Surg 2007;23(2):69-72.

7. Ozeren S, Caliskan E, Corakci A, Ozkan S, Demirci A. Magnetic resonance imaging and angiography for the prerupture diagnosis of rudimentary uterine horn pregnancy. Acta Radiol 2004;45:878-881.

8. Cunningham FG, MacDonald PC, et al, editors. Williams obstetrics, 19th ed. New Jersey: PrenticeHall International Inc; 1993. pp. 543-53.
9. Desai BR, Patted SS, Pujar YV, et al. Advanced secondary abdominal pregnancy following rupture of rudimentary horn. $\mathbf{J}$ Obstet Gynecol India 2005;55:180.

10. Heinonen P. K. Complete septate uterus with longitudinal vaginal septum. Fertil Steril. 2006; 85(3): 700-5.

DOI: $10.5455 / 2320-1770 . i j r \operatorname{cog} 20131258$

Cite this article as: Gupta N, Gupta S, Singh N, Arya AK, Lal P, Chauhan M. A rare case of uterus didelphys presented as secondary abdominal pregnancy. Int J Reprod Contracept Obstet Gynecol 2013;2:737-9. 